\title{
THE DEFLECTIVE EFFECT OF THE EARTH'S ROTATION.
}

By Charles F. Brooks, Instructor in Geography, Yale University, New Haven, Conn.

Halley, the famous English astronomer, in 1686 tried to explain the oblique course of the trade winds as a result of their following the diurnal heat wave which accompanies the (apparent) westward movement of the sun around the earth. It was not until 1735 , however, that the basic cause for the deflection of the winds was discovered. In that year, Hadley advanced the theory that winds with a north or south component are deflected to the west or east because they move into latitudes having increasing or decreasing rotational velocities, respectively. It was assumed that the winds merely take with them the rotationa' velocity of the latitude whence they come. The correct explanation was advanced first by Charles Tracy in 1843; but the more complete work of William Ferrel beginning in 1856 received wider recognition. The following exposition is taken mostly from Ferrel's "A Popular Treatise on the Winds" (New York, 1889), pages 42 to 88. Professor W. M. Davis has rendered an excellent nonmathematical account in his "Elementary Meteorology" (Boston, 1894), pages 101 to 108 . Furthermore, Dr. Julius von Hann has published a full bibliographic summary of the earth's deflective effect, in his "Lehrbuch der Meteorologie" (Leipzig, 1915), pages 430 to 434 .

Professor Davis's introductory paragraph on deflections runs as follows:

"The cause of the deflection of the winds from the gradients is to be found in the earth's rotation. It may be easily explained and illustrated by experiment (see pp. 106-108) that the winds cannot follow the gradients, because there arises from the earth's rotation a.force that tends to deflect all horizontal motions, of whatever direction, to the right in the northern hemisphere, and to the left in the southern: the deflecting force is proportionate to the velocity of motion, and increases from zero at the equator to a maximum value at either pole." A footnote on centrifugal force adds: "Although always spoken of as a'force,' this term implies a misconception of the same kind as that which often embarrasses the understanding of 'centrifugal force.' A body moving without friction over the surface of the earth tends to move in the direction of its first impulse. We live on the earth's surface, unconscious of its rotary movement, and consequently persuaded that any straight line holds a fixed direction. Hence, when a free- 
moving body (such as a free-swinging pendulum, as in Foucault's experiment) turns aside from its first fine of movement, we assume that its direction has been changed by some deflecting force. In reality, the free-moving body perseveres in its original direction, in virtue of its inertia; it is the apparently fixed line of reference that is changing its direction, in virtue of the earth's rotation. The "deflecting force" is therefore only the inertiaresistance that a free-moving body exerts against a constraining force that urges it to move in what we call a straight line or a fixed direction."

Stating this differently, Ferrel says (p. 79): "Some general idea of this deflecting force may be formed from the experience of any one in walking over a narrow drawbridge while it is turning around its central pivot. If there were no railings, the tendency would be, if not guarded against, to run off on the one side or the other, according to the direction of gyration; and where there are railings, to press against that side. And this tendency would evidently be in proportion to the velocity of transit across the bridge and the angular velocity of its gyration. Of precisely the same nature is the deflecting force of the earth's rotation; for every horizontal line, fixed relatively to the earth's surface, except at the equator, is continually changing its direction with reference to a direction fixed in space; and so a body, set in motion in any direction relative to the earth's surface, tends, if free, to depart from this direction, and if constrained to move as in a groove in his direction, to press toward one side or the other, as the case may be."

That a rotating table may be employed to illustrate the deflection, may be made clear by the following illustration (Davis, p. 108):

"Several circular dises of paper, an inch or two in diameter and each marked with a strong diametral line, may be attached to a terrestrial globe in different latitudes. Watch the diametral line on one of the discs while the globe is slowly rotated; the line will be seen to change its direction; now pointing to one part of the room, now to another. In other words, the dise is rotating with respect to its center, and in the same direction as the globe rotates. A dise near the pole will rotate rapidly; a dise near the equator will turn its diameter more slowly from one direction to another; a dise on the equator has no motion of rotation with respect to its center; and at the equator there is no deflective force." 
The angular rate of rotation of any disc is equal to the angular rate of rotation of the globe multiplied by the sine of the latitude. So, as the earth's rotation rate is constant, the angular rate of deflection of any moving body at a given latitude is constant, and is the same irrespective of the direction of motion of the body. But the actual distance of deflection in any given time depends on the body's velocity. This departure of the body from the initial direction for a very short range may be expressed by the following formula (Ferrel, p. 86):

$$
d=0.00007292 s \sin l . t^{2}
$$

in which $d$ is the departure in meters; 0.00007292, the angular velocity of the earth's surface expressed in terms of the radius; (Ferrel, p. 62); $l$ is the geographical latitude; $s$, the velocity in meters per second; $t$, the time in seconds.

The foregoing shows why there is deflection. The forces acting to produce it deserve further explanation. Any body on the earth is held down by gavity so that even when "at rest" it is travelling around the axis of rotation at a high speed, except in the immediate vicinity of the poles. If the earth were spherical, gravity, being equal everywhere, could not prevent the body from sliding equatorward. But it does not move equatorward because this tendency has already built up a bulge such that the pull of gravity down the slope just counterbalances the centrifugal tendency up the slope ${ }^{1}$.

If this body or mass of air is set in motion eastward its total rotative velocity around the earth's axis becomes greater. The centrifugal force is thus increased: therefore, the body moves towards the equator, up the unchanged slope. On the other hand, the body, when given a westward motion relative to the earth's surface, suffers a diminution of its total rotative velocity. The centrifugal force, consequently, becomes insufficient to counterbalance the gravitative action and the body moves down the slope, poleward. Both of these deflections are to the right in the northern hemisphere and to the left in the southern. - A vertical component of the centrifugal force tends to deflect west winds upward and east winds downward.

\footnotetext{
${ }^{1}$ In general the centrifugal force $=F_{c}=\frac{\omega}{r}^{2} m$ (Ferrel p. 47), in which $\omega=r n=$ the gyratory velocity in terms of the radius, $r$ being the radius vector and $n$ the angular velocity: $m$ stands for mass.

Applied to the earth this formula becomes:

$\mathrm{F}_{\mathrm{e}}=2 n s \sin l . m$

(Ferrel p. 81),

where $n=2 \frac{\pi}{86164}=0.00007292=$ the angular velocity of the earth's surface expressed in terms of the radius; $s=$ the velocity of the body; and $l$. = the geographical latitude.
} 
A body in motion equatorward is going farther from the axis of rotation. According to Kepler's second law, the radius vector describes equal areas in equal times. Thus when the radius increases, the angular velocity must decrease. But the surface of the earth at all points has the same angular velocity; and, therefore, the body in motion equatorwards is apparently deflected to the west. If the body goes poleward, on the contrary, the radius vector becomes shorter; and the angular velocity greater. The result in both cases is a deflection to the right in the northern hemisphere and to the left in the southern hemisphere.

As movements in any direction can be resolved into their meridional and latitudinal components, it is evident that there is deflection to the right in the northern and to the left in the southern hemisphere no matter in which way the body moves; a deflection which, as previously stated, is equal for all directions. ${ }^{2}$

Ferrel compares his results with Hadley's as follows (pp. 68-69):

"The preceding results, obtained from the principle of 841 [Kepler's principle of the preservation of areas], differ very much from those obtained form the principle adopted by Hadley, about the year 1735, in explaining the trade-winds, namely, that a body, being forced directly toward or from the pole, tends to keep its initial absolute gyratory velocity. He says:" 'A particle of air drawn from the tropies, where it is supposed to have no motion east or west, toward the equator acquires a westward velocity on account of the parallels continually enlarging. The increase of the parallels from the tropics to the equator is in the ratio of 917 to 1,000 , and hence the westward motion in an hour is 83 miles at the equator, which is decreased by the effect of the earth's surface to what is observed.' But from what has been shown above, the velocity of a body having an absolute east velocity of 917 miles per hour at the tropics, as here supposed, would be decreased at the equator in the ratio of unity to the cosine of the latitude at the tropics, or as 1 to 0.917 . Hence, instead of still having an absolute east velocity of 917 miles per

\footnotetext{
2This equality Ferrel proves mathematically, arriving at these three formulas:

$\mathrm{Fu}=(2 n+-\sim) u \sin l m=$ eastward deflecting force with poleward velocity $u$.

$F_{\mathbf{v}}=(2 n+\sim) v \sin l m=$ equatorward deflecting force with east ward velocity $v$

i's $=(2 n+\sim) s$ sin $l m=$ force deflecting body to right in northern hemisphere when $s$ is the velocity of movement in any direction along the earth's surface. $n=$ the absolute gyratory velocity; and $v=$ the relative gyratory velocity of the body with mass $m$ at geographical latitude $l$ (pp.69-73).

"This deflecting force, however, is not a real force, but is of the same nature as centrifugal force, $\$ 35$; and as it always acts, as we have just seen, in a direction at right angles to the direction of motion, its tendency is to continually change direction only, and not to increase or decrease velocity and momentum. But this change of direction must not be understood to be a change of absolute direction in space, for this would require a real force, but simply a change of direction relative to the earth's surface" (p. 73)

${ }^{3}$ Concerning the Cause of the General Trade-Winds; by Geo. Hadley, Esq. Phil. Trans., 1735.
} 
hour, it would have a velocity of only $917 \times 0.917=841$ miles per hour. This being deducted from 1,000, the supposed absolute velocity of the earth's surface at the equator, we have 159 miles for the relative east velocity there, instead of 83 miles as given above.

"According to this erroneous principle, in order to obtain the relative east components of velocity of the body at the several latitudes, in moving from the equator to the pole in the preceding example, it would be necessary to subtract the absolute east velocities of the several parallels, as given in Table $\mathrm{V}$, from that of the equator, and we should thus get in meters per second, at the parallel of $45^{\circ}, v=465-329=136$ [Ferrel: $658-329$ $=329$ ]; at the parallel of $60^{\circ}, v=465-232=233$ [Ferrel: $930-232=698] ;$ and at the parallel of $80^{\circ}, v=465-81$ $=384$ [Ferrel: $2674-81=2593$ ]." Of course, friction and, with gases and liquids, mixing and other factors prevent the occurrence of such enormous velocities due to the earth's rotation.

Hadley's theory applied to east and west winds requires no deflection of these winds, while as a matter of fact, deflections are the same irrespective of the wind direction. Another point not mentioned by Ferrel is that if from either pole a body moves without friction along the earth's surface, the deflection according to Hadley's theory is correct, for the body has no rotational velocity. It is little wonder that Hadley's theory with its simplicity and partial truth should still be accepted as correct. ${ }^{4}$

Summary.

The apparent deffection of bodies moving on the earth's surface occurs because the earth turns in space while the body tends to travel in a straight line. The angular rate of deflection varies with the latitude, but is the same for motion in any direction at a given latitude. The absolute distance of deflection depends, in addition, on the velocity of the body in motion. Stated in terms of the forces involved, an eastward or a westward movement respectively increases or decreases the centrifugal force. The equilibrium between gravity and centrifugal force as maintained by the equatorial bulge is thus disturbed, and deflection results. A movement equatorward or poleward so changes the rotational velocity of the body that it is deflected to an extent equal to the deflection with eastward of westward motion. In fact, for all directions of horizontal movement there is deflection to the right in the northern hemisphere and to the left in the southern hemisphere.

4For instance, see: E. C. Martin, Our Own Weather, New York, 1913, p. 23. 